\title{
Frequency-Response Coupling between Synchronous Areas in
}

\section{Europe}

\section{Conference Paper}

\section{Author(s):}

Scherer, Marc; Zolotarev, Pavel

Publication date:

2015

Permanent link:

https://doi.org/10.3929/ethz-b-000105093

Rights / license:

In Copyright - Non-Commercial Use Permitted

Originally published in:

https://doi.org/10.1109/PTC.2015.7232681 


\section{Frequency-Response Coupling between Synchronous Areas in Europe}

\author{
Marc Scherer \\ Swissgrid Ltd., Switzerland \\ marc.scherer@swissgrid.ch
}

\author{
Pavel Zolotarev \\ TransnetBW GmbH, Germany \\ p.zolotarev@transnetbw.de
}

\begin{abstract}
This paper investigates different control implementations for the frequency-response coupling between synchronous areas that feature a decentralized load-frequency control structure. The technical basics are presented, along with a 2-area benchmark system implemented in SIMULINK. Two new concepts are applied: Frequency-response delivery and frequency netting. The results show that both concepts are robust and stable. But as the frequency-response coupling affects the frequency performance in both the connecting and receiving synchronous area, the control scheme determines the overall system reliability. There are two reasonable options for the unilateral frequencyresponse delivery; the choice depends on the difference in the frequency restoration design between the synchronous areas. For frequency netting, only one scheme is applicable.

Index Terms-Frequency containment reserves, frequency netting, frequency-response, primary frequency control.
\end{abstract}

\section{INTRODUCTION}

The successful operation of interconnected power systems requires real-time balancing of generation and consumption. Therefore, frequency control represents an important part of ancillary services and is crucial for the security of supply. However, the exchange of ancillary services between synchronous areas is mostly limited to mutual emergency assistance [1]. To our knowledge, there are no studies with a systematic approach for the frequency-response coupling between synchronous areas within ENTSO-E, where technical standards in particular are of interest in the discussion of panEuropean ancillary service markets [2], [3].

So far, Sterpu and Tuan [4] analyzed HVDC substations from a frequency control provision point of view and quantified their potential performance. Fujita et al. [5] as well as Sanpei et al. [6] discussed the possibility of reducing load fluctuation by asynchronously interconnecting isolated power systems in Japan. For island systems being connected to large interconnected power systems such as Tasmania and Australia, there are already HVDC control and protection systems in use that act upon the frequency difference in the systems [7].

In this paper, we focus on the technical frequency-response coupling and its impact on the frequency performance in both the providing and the receiving system as the precondition for an economic optimization of frequency-response reserve procurement between synchronous areas. We investigate the technical possibilities for frequency-response delivery as well as frequency netting between synchronous areas. Our contribution is to specify possible control implementations for the frequency-response coupling between different synchronous areas and to demonstrate the basic effects which are independent of the parameters.

This paper is organized as follows: Section II elaborates the frequency control basics for investigating a potential frequency-response coupling. Section III outlines the modeling. Section IV presents the results and discusses the implications on system security. Finally, Section V is devoted to conclusions and perspectives.

\section{Frequency Control Basics}

\section{A. Current Three-Tiered Approach}

In most interconnected power systems, frequency control is a three-tiered approach; the associated capacities are referred to as active power reserves or control reserves [8], [9]: Frequency Containment Reserves (FCR) are a joint responsibility distributed among all Transmission System Operators (TSOs) in a Synchronous Area (SA). The control structure is proportional; thus, a steady state error is unavoidable. Frequency Restoration Reserves (FRR) imply a local responsibility of each TSO for the imbalance in its control area. Frequency restoration can be performed by either automatic reserves (see Section II-B) or fast-acting manual reserves. Replacement Reserves (RR) are manual reserves featuring an activation time longer than the time to restore frequency; hence, they are meant to supplement FRR, e.g. after a large incident.

\section{B. Automatic Generation Control}

In large power systems such as Continental Europe or the IPS/UPS interconnection, the frequency restoration responsibility is shared among various control areas by means of Automatic Generation Control (AGC). The AGC principles are based on the fact that the quasi-steady state frequency is the same in the entire synchronous area; therefore, decentralized feedback implemented by each control area for responding to the local imbalance contributes to the overall balance in the system. The Area Control Error $A C E$ is the sum of the weighted frequency deviation and the deviations of the net tieline flow between the control area and its neighbors. The $A C E$ is the control error for a PI controller with anti-windup; its control signal $P_{\mathrm{AGC}}$ is sent to reserve providing units, which deploy the respective amount of active power reserves, i.e. FRR. If the frequency bias factor $K$ is chosen appropriately, a control area will only compensate for its imbalance, and it will 


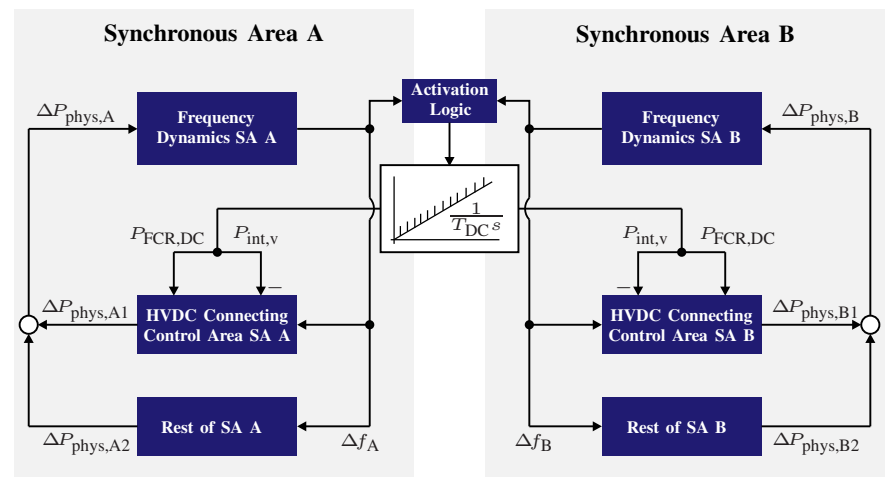

(a) Two synchronous areas with frequency-response coupling.

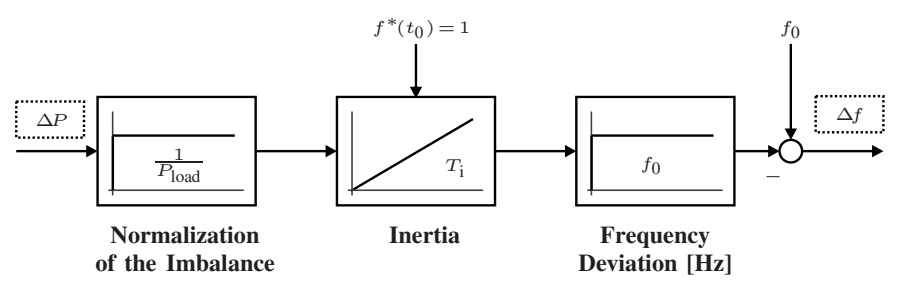

(b) The frequency dynamics.

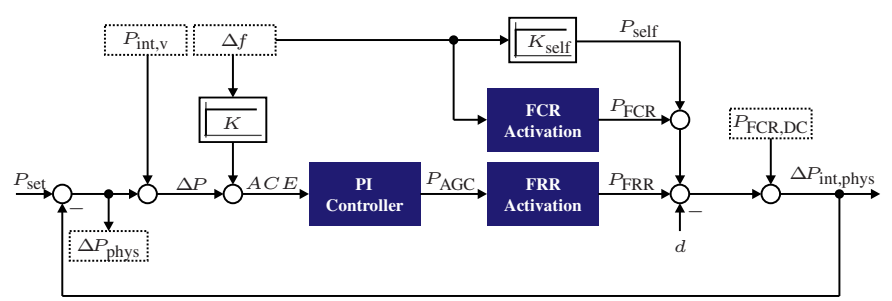

(c) The control area model including frequency containment, self-regulation and AGC.

Fig. 1: Visualization of the simulation framework.

neither counteract its frequency containment contribution nor its share of self-regulation; vice versa, AGC will compensate for a non-delivery of FCR in the respective area. This principle is referred to as non-interactive control [9].

\section{Modeling}

\section{A. Simulation Framework}

The investigation of frequency-response coupling between synchronous areas is based on a simulation framework, which describes the dynamic frequency behavior of a synchronous area including the automated control processes for its stabilization. The simulation framework is illustrated in Figure 1.

The model consists of two synchronous areas $\mathrm{A}$ and $\mathrm{B}$ connected by a HVDC link as shown in Figure 1a. The flow on the HVDC link is adjusted by an activation logic which depends on the frequency-response implementation.

A synchronous area model includes two main components:

- a frequency dynamics model with the active power imbalance of the synchronous area as input and its frequency deviation as output as well as

- a control area model which describes the frequency containment and restoration dynamics.
Figure $1 \mathrm{~b}$ shows the frequency dynamics model which describes the relationship between an active power imbalance, the inertia and the frequency deviation. Figure 1c shows the control area model which describes its frequency response consisting of FCR activation and the self-regulation effect as well as the AGC loop including the $A C E$ calculation and the respective FRR activation. The disturbance $d$ (e.g. a power plant outage) affects the actual power interchange of the control area $P_{\text {int,phys }}$, which is compared to its scheduled power interchange $P_{\text {set }}$. The resulting value $\Delta P_{\text {phys }}$ corresponds to the physical power imbalance of the control area and is applied to the input of the frequency dynamics model.

The flow on the virtual tie-line $P_{\text {int,v }}$ is also part of the $A C E$ calculation and is a control signal which is typically updated in the time frame of seconds and serves an offset to the input of the PI controller. The term "virtual" is used in ENTSO-E terminology to distinguish this calculated part of the $A C E$ from physically measured tie-lines. In this paper, the virtual tie-line is used for some of the frequency-response coupling methods. It is worth noticing that the term $K \Delta f$ can be seen as a special case of a virtual tie-line. The frequency-response coupling between the synchronous areas via the HVDC link is represented by the signal $P_{\mathrm{FCR}, \mathrm{DC}}$ which influences the power interchange in the same way as a disturbance.

\section{B. Implementation of Frequency-Response Coupling}

The frequency-responses of the synchronous areas must be coupled by the means of a HVDC link. In this context, there are several options for the integration of the HVDC power flow into the AGC loop, in particular by use of virtual tie-lines.

The simulation framework according to Figure 1 is used to model the possible implementation schemes for a frequencyresponse coupling:

- Scheme 1: The HVDC flow has only a physical impact on the $A C E$ calculation of both control areas $\left(P_{\text {int, } \mathrm{v}}=0\right)$.

- Scheme 2: The HVDC flow is added to the $A C E$ calculation of the HVDC connecting control area of the synchronous area which requests a frequency-response $\left(P_{\text {int }, \mathrm{v}}=P_{\mathrm{FCR}, \mathrm{DC}}\right.$ in $\left.\mathrm{SA} \mathrm{B}\right)$.

- Scheme 3: The HVDC flow is subtracted from the $A C E$ calculation of the HVDC connecting control area of the synchronous area which provides a frequency-response $\left(P_{\text {int }, \mathrm{v}}=-P_{\mathrm{FCR}, \mathrm{DC}}\right.$ in $\left.\mathrm{SA} \mathrm{A}\right)$.

- Scheme 4: Combination of Scheme 2 and Scheme 3.

\section{Implementation of Frequency Netting}

Frequency (deviation) netting can be considered a special case of frequency-response coupling. In this case the HVDC flow is adjusted based on a logic which compares the signs of the frequency deviations in the synchronous areas in order to avoid an opposing FCR activation. Whenever one synchronous area is short and the other is long, the HVDC flow is adjusted in such a way that the imbalances in the synchronous areas are offset and the counteracting FCR activation is reduced. The term frequency netting is used in order to indicate the 
difference to imbalance netting, which is a concept to avoid opposing FRR activations [10].

\section{RESUlts}

The synchronous areas are parameterized according to the dynamic behavior of Continental Europe [8]. The flow over the HVDC is always adjusted proportionally to the frequency deviation with a static of $5 \mathrm{GW} / \mathrm{Hz}$ (the total flow is limited to $1 G W)$.

To assess the different technical implementations, we analyze the frequency control dynamics of a standardized incident, i.e. the N-2 reference incident, which is $3 G W$ for Continental Europe. First, we analyze the four technical schemes for the main purpose of delivering FCR from SA A to SA B. Second, we evaluate the same schemes for frequency netting.

\section{A. Frequency-Response Delivery}

In minute one the SA B is disturbed, which immediately leads to a frequency deviation and the activation of FCR in the entire synchronous area. The HVDC flow leads to an imbalance in the SA A causing a frequency deviation which leads also to a FCR activation in the other synchronous area.

In Scheme 1, the frequency-response coupling is neither considered in the AGC loop of SA A nor in the one of SA B. Figure 2 illustrates the dynamic behavior, if the disturbance is located in the HVDC connecting area of SA B. Both HVDC connecting control areas trigger AGC and activate FRR in order to compensate the local imbalance resulting from the FCR delivery, which will bring the frequencies back to $50 \mathrm{~Hz}$. With the decreasing frequency deviation in SA B the need for FCR activation is also reduced and the frequency-response delivery from SA A via the HVDC decreases. This leads to a decreasing imbalance in SA A. The already activated FRR in SA A need to be deactivated, which is only possible with a delay inherent to the dynamics of the AGC loop of the HVDC connecting control area. Therefore, there is a time frame, roughly between 3.5 and 10 minutes, when there is too much power in the SA A and, thus, a negative frequency deviation. Approximately after 14 minutes the imbalance is controlled in both synchronous areas and the HVDC flow is zero again. However, this only applies if the HVDC connecting area has enough AGC resources, i.e. FRR, to cope with the imbalance in steady state. If the amount of FRR in the HVDC connecting area falls below the magnitude of the imbalance, a steady state frequency deviation is unavoidable in SA B. The HVDC flow will not return back to zero, and the respective amount of FRR will be activated in the HVDC connecting area in SA A.

Moreover, Scheme 1 is disadvantageous, if the disturbance does not originate in the HVDC connecting area in SA B. In this situation, SA A similarly supports SA B, but the AGC of the HVDC connecting area of SA B will counteract the FCR delivery by automated FRR activation. Figure 3 shows the dynamic behavior after a disturbance located outside the HVDC connecting area in SA B. The frequency characteristic

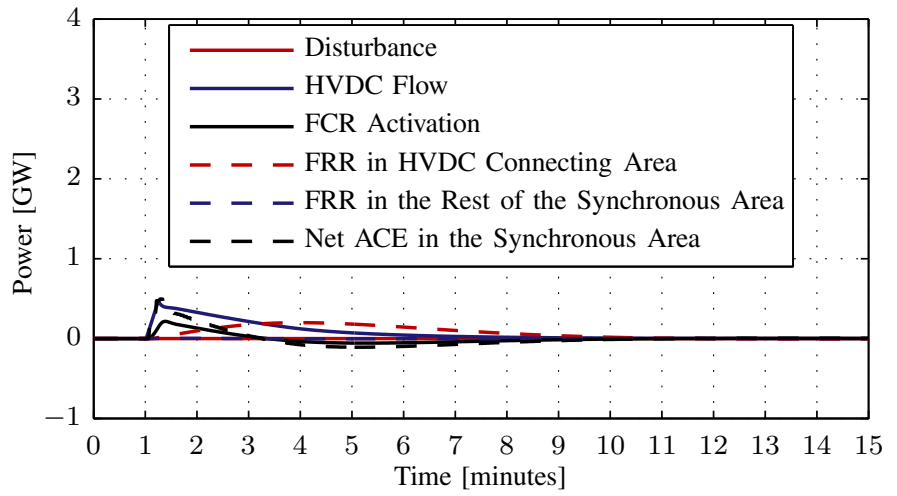

(a) The providing synchronous area.

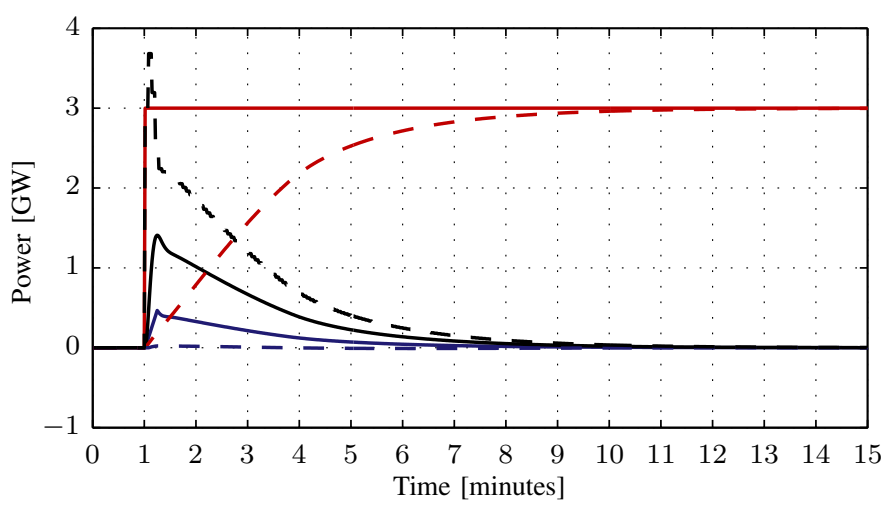

(b) The receiving synchronous area (same legend as in Figure 2a).

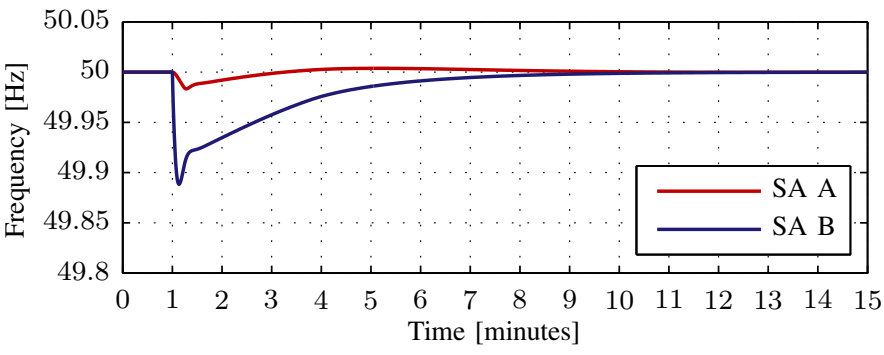

(c) Frequencies in both synchronous areas.

Fig. 2: Scheme 1, the HVDC connecting area in SA B is disturbed and the amount of FRR is not limited.

is similar but slower than in Figure 2. Therefore, the applicability of Scheme 1 is very limited, and the dynamic response is slow compared to other schemes.

In Scheme 2, the additional HVDC flow is considered in the AGC loop of SA B. With that, the dynamic response is not only faster as in Scheme 1 but also generally applicable, as AGC in the HVDC connecting area does not counteract the HVDC flow. The overall dynamic behavior for a disturbance inside the HVDC connecting area looks similar as for Scheme 1. The HVDC flow consideration implies a consistent $A C E$ calculation: In case of limited FRR in the concerned area in SA B, SA A activates AGC, i.e. the steady state frequency deviation is always zero in SA A. Thus, Scheme 2 is robust, but in steady state not only FCR will be provided as AGC 


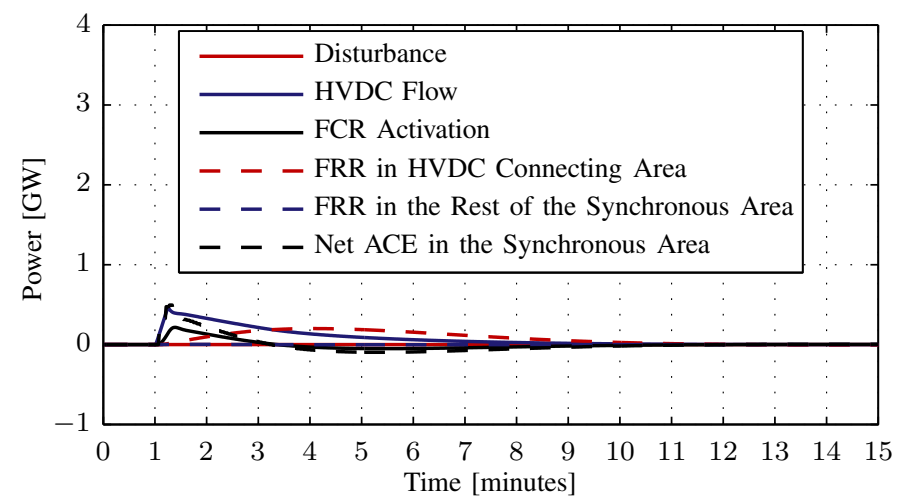

(a) The providing synchronous area.

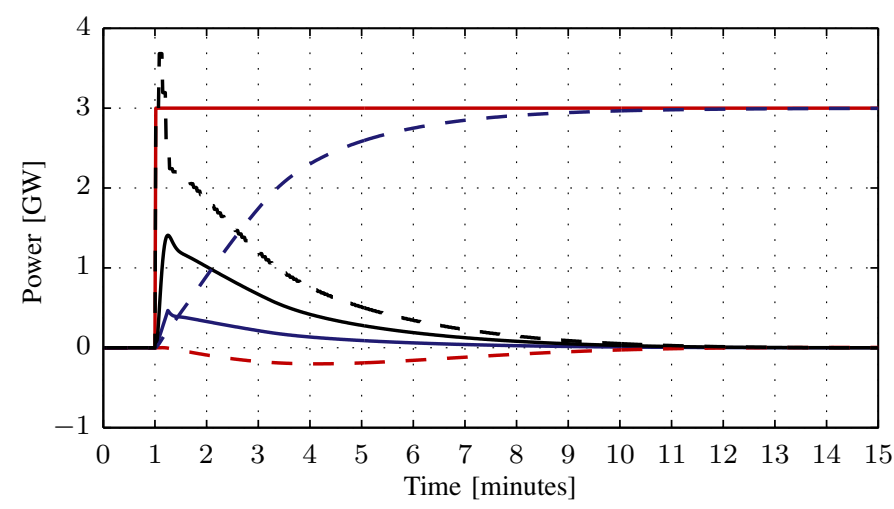

(b) The receiving synchronous area (same legend as in Figure 3a).

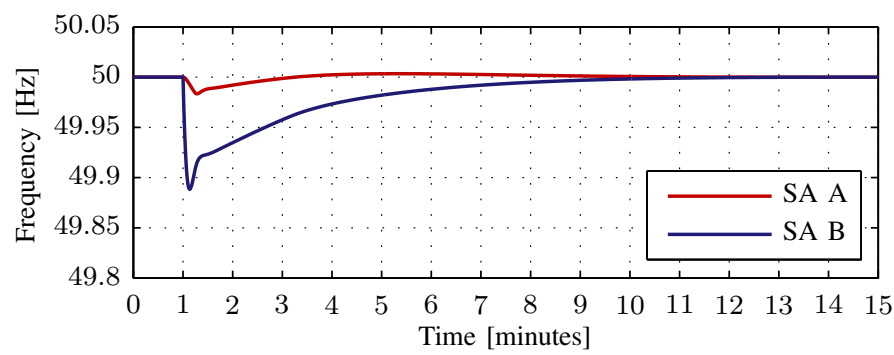

(c) Frequencies in both synchronous areas.

Fig. 3: Scheme 1, the disturbance does not occur in the HVDC connecting area in SA B and the amount of FRR is not limited.

is triggered if reserves are exhausted in the area where the disturbance occurs in SA B.

Scheme 3 offers the possibility to avoid the AGC activation in steady state in SA A by including the HVDC flow into the AGC loop of SA A. If FRR are limited in the concerned area, there will be a steady state frequency deviation in both synchronous areas. However, Scheme 3 harks back to the problem of counteracting AGC (as already depicted in Figure 3), if the disturbance is not located in the HVDC connecting area of SA B; thus, Scheme 3 exhibits identical shortcomings as Scheme 1 and should, therefore, be avoided for the same reasons.

Scheme 4 is the subsequent combination of Scheme 2 and Scheme 3. It features the advantages of both: Independent of

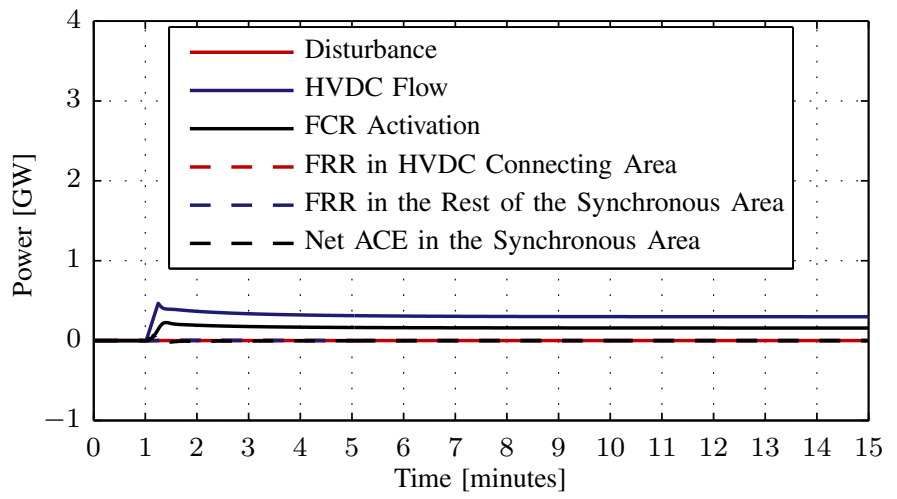

(a) The providing synchronous area.

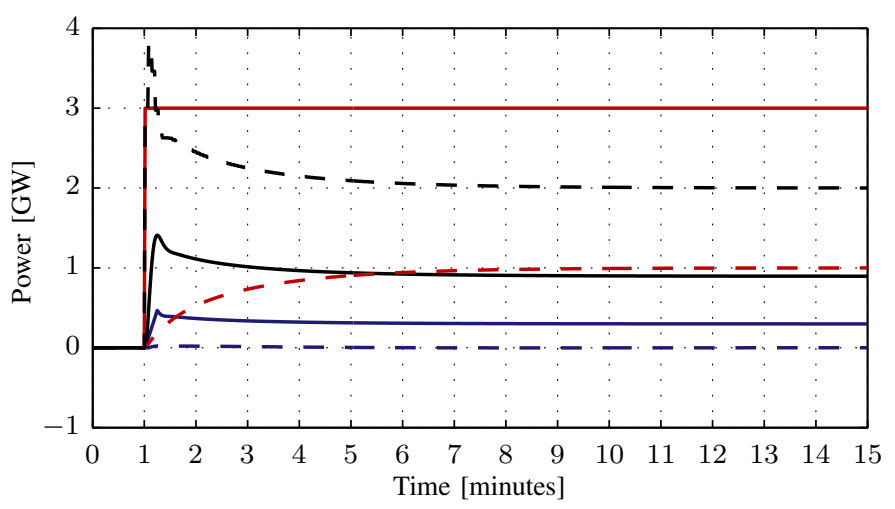

(b) The receiving synchronous area (same legend as in Figure 4a).

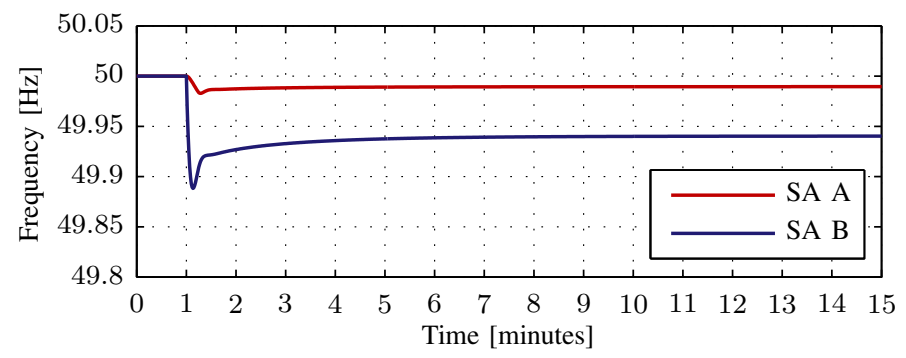

(c) Frequencies in both synchronous areas.

Fig. 4: Scheme 4, the HVDC connecting area in SA B is disturbed and the amount of FRR is limited to $1000 \mathrm{MW}$.

the disturbance's origin, AGC does not counteract the HVDC flow, and no AGC is triggered in steady state in case of a shortage of FRR in SA B. The dynamic behavior for this setup is shown in Figure 4. Therefore, Scheme 4 provides for the most efficient implementation. However, with Scheme 4, the possibility to monitor performance compliance based on $A C E$ measurements is more limited, as an overall $A C E$ of zero in SA A does not necessarily imply adherence to the set point frequency.

To summarize, only Scheme 2 and Scheme 4 are technically robust implementation approaches which do not necessarily deteriorate the frequency quality in one or both of the areas. Table I shows a qualitative assessment of the four options regarding the resulting interaction with AGC. 


\begin{tabular}{llll}
\hline & $\begin{array}{l}\text { SA A } \\
\text { (FRR of SA B is } \\
\text { Sufficient) }\end{array}$ & $\begin{array}{l}\text { SA A } \\
\text { (FRR of SA B is } \\
\text { Exhausted) }\end{array}$ & SA B \\
\hline Scheme 1 & Neutral & Neutral & Deteriorating \\
Scheme 2 & Neutral & Neutral & Neutral \\
Scheme 3 & Neutral & Deteriorating & Deteriorating \\
Scheme 4 & Neutral & Deteriorating & Neutral \\
\hline
\end{tabular}

TABLE I: Qualitative impact of the four schemes on the frequency quality.

Scheme 4 can lead to a worsening of the frequency quality, either if not enough reserves are available in the concerned control area or if the time to restore frequency is significantly higher in SA B than in SA A. In such a situation, Scheme 2 is preferable as it ensures a zero steady state frequency within the design parameters of the synchronous areas. However, an evaluation of the $A C E$ quality of the HVDC connecting TSO is than affected by the AGC performance of SA B; thus, Scheme 2 is the safest implementation from an operational point of view, but rather inconsistent compared to a FCR activation within the synchronous area.

\section{B. Frequency Netting}

Frequency netting is a reciprocal service in order to make use of opposing frequency deviations between the synchronous areas. To investigate the effects of frequency netting, a disturbance occurs in both synchronous areas, but in opposite directions: In minute one the SA A is disturbed by a loss of production of $3 G W$, followed by a loss of load of $2 G W$ in SA B $40 s$ later.

The dynamic behavior of frequency netting with Scheme 1 is presented in Figure 5. As demonstrated in Section IV-A, Scheme 1 leads to an activation of FRR in the HVDC connecting area in order to compensate the disturbance created by the HVDC flow. This leads to a counteracting FRR activation in the disturbed and the HVDC connecting areas in SA A and SA B in steady state; thus, frequency netting is not turned to advantage.

Scheme 2 and Scheme 3 have a similar behavior. They will lead to a one-sided effect as only one area includes the imbalance as a virtual tie-line exchange. Obviously, Scheme 4 allows for a reciprocal frequency netting; with this scheme, it is possible to take full advantage of frequency netting as illustrated in Figure 6.

Table II illustrates that only Scheme 4 allows to take full advantage of frequency netting.

\begin{tabular}{lll}
\hline & SA A & SA B \\
\hline Scheme 1 & No & No \\
Scheme 2 & No & No \\
Scheme 3 & No & No \\
Scheme 4 & Yes & Yes \\
\hline
\end{tabular}

TABLE II: Applicability of the different schemes for frequency netting.
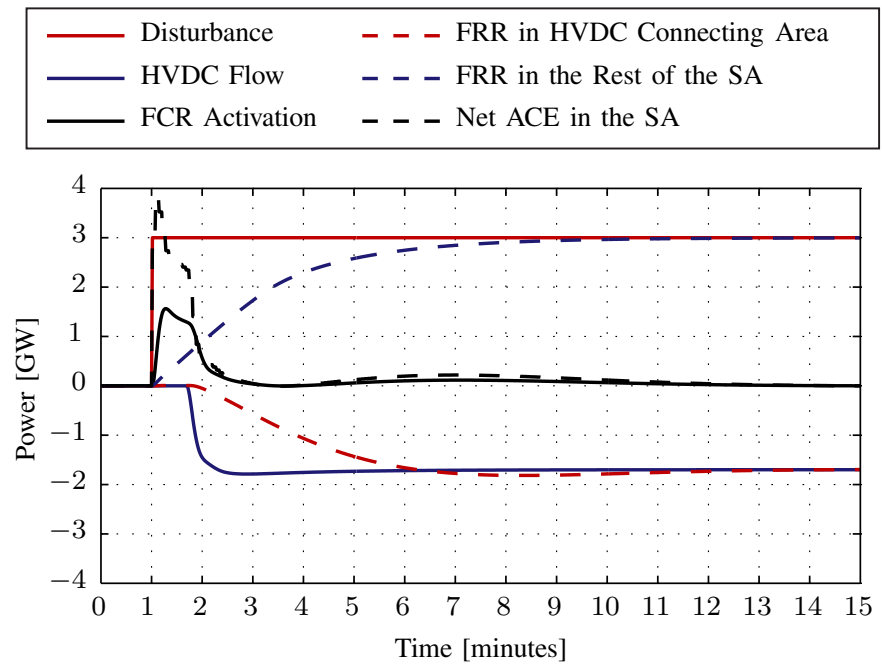

(a) The providing synchronous area.

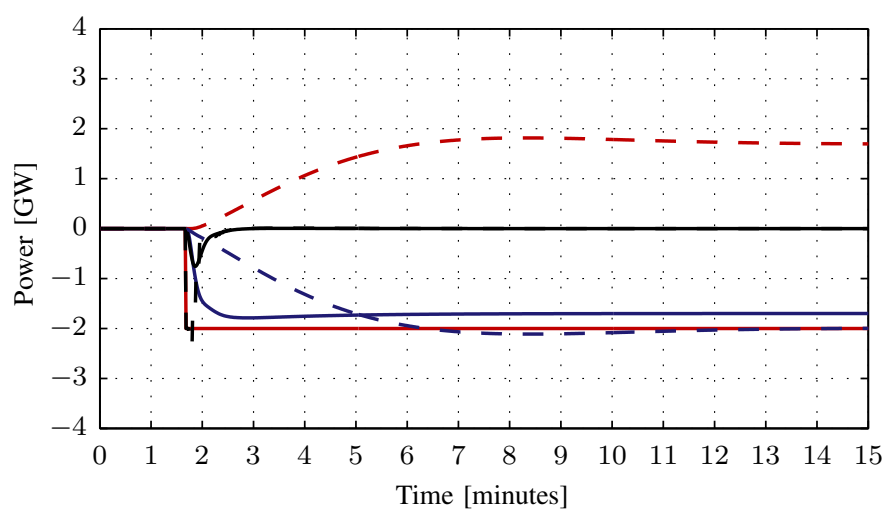

(b) The receiving synchronous area (same legend as in Figure 5a).

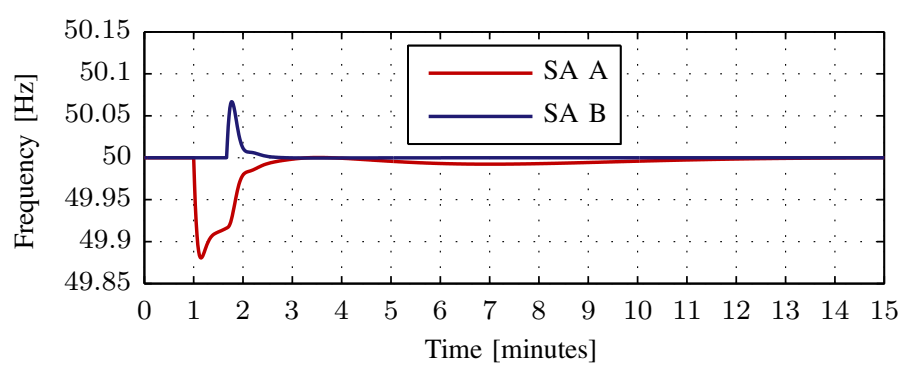

(c) Frequencies in both synchronous areas.

Fig. 5: Frequency netting with Scheme 1, the disturbance does not occur in the HVDC connecting area.

\section{Closing Remarks}

\section{A. Conclusion}

The paper demonstrates how frequency-response coupling can be implemented between synchronous areas that feature a decentralized load-frequency control structure. For the integration of the frequency-response delivery into the AGC loops, the use of a virtual tie-line in the receiving synchronous area is recommended in order to include the FCR delivery from the other synchronous area in the $A C E$ calculation in the same way as flows due to FCR from the same synchronous area. The 


\begin{tabular}{|c|c|}
\hline $\begin{array}{ll} & \text { Disturbance } \\
& \text { HVDC Flow } \\
\text { FCR Activation }\end{array}$ & $\begin{array}{l}\text { - - - FRR in HVDC Connecting Area } \\
\text { - - - FRR in the Rest of the SA } \\
\text { - - - Net ACE in the SA }\end{array}$ \\
\hline
\end{tabular}

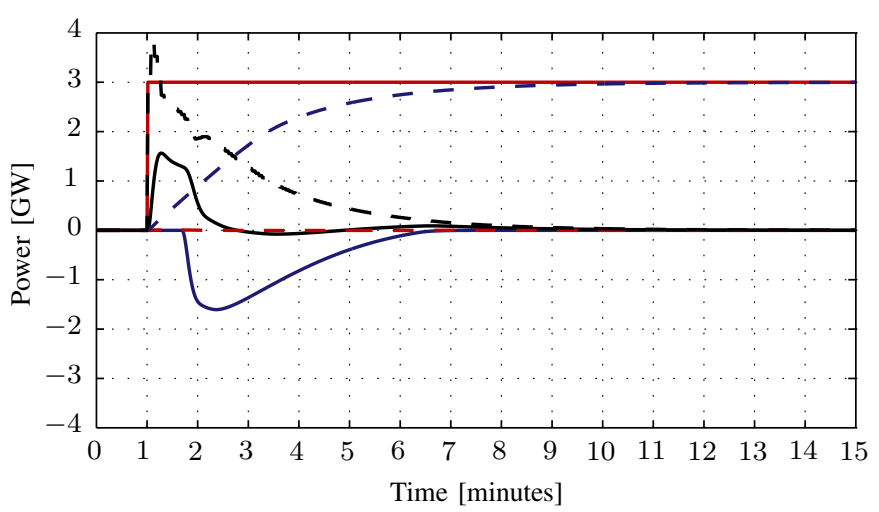

(a) The providing synchronous area.

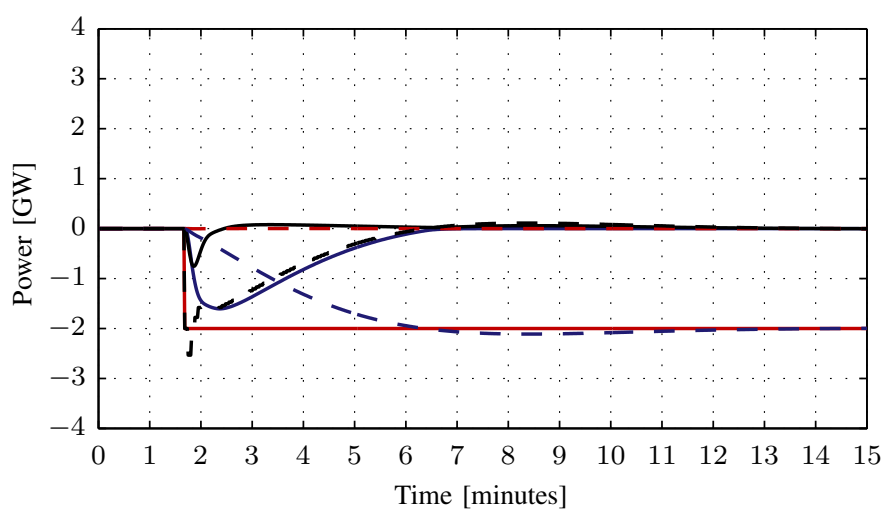

(b) The receiving synchronous area (same legend as in Figure 6a).

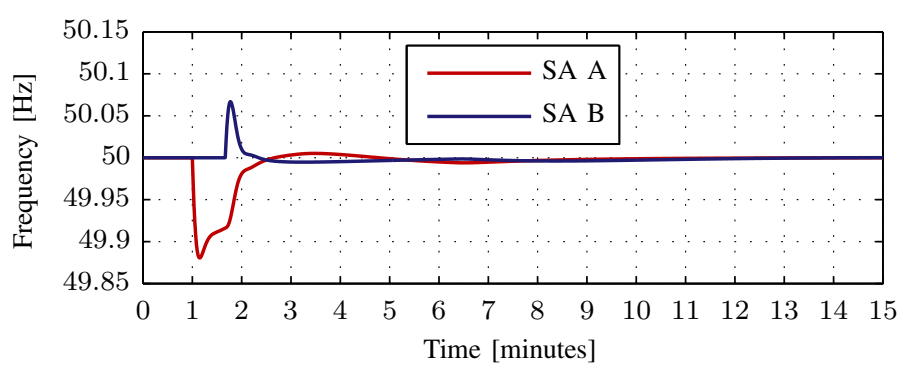

(c) Frequencies in both synchronous areas.

Fig. 6: Frequency netting with Scheme 4, the disturbance does not occur in the HVDC connecting area.

HVDC connecting control area of the providing synchronous area will activate FRR in case of FCR delivery to another synchronous area. This can be prevented by using a virtualtie line in order to include the FCR delivery in the $A C E$ calculation as an intended flow, as it is the case for the same synchronous area. In this case, not only the frequency-response of FCR but also the frequency restoration processes of the synchronous areas will be coupled and there is a need for at least a partial harmonization of the related parameters and quality targets.
Frequency netting is a possibility to couple the frequencyresponse only if no additional imbalances are created. In this case virtual tie-lines need be used in order to prevent a counteraction of frequency netting by the AGC loops of the HVDC connecting control areas.

\section{B. Outlook}

For a large-scale frequency-response coupling between synchronous areas, not only the technical implementation needs to be considered but also the ancillary service procurement. However, a synchronous area coupling can be extended to FRR, which are activated based on a set-point provided by a TSO. With this, it is possible to activate a dedicated provider for another synchronous area without inducing a frequency deviation in the providing area.

In that context, the European work in progress "Guidelines" and "Network Codes" should facilitate a regulatory framework including the limits of the applicability of the economic rationale, as the discussed schemes can be used for both the sharing and the exchange of reserves. Exchange means that a part of the active power reserves is located in another synchronous area, i.e. the total amount remains the same as without an exchange. In case of sharing, the overall amount is reduced based on the assumption that disturbances in both synchronous areas are not strongly correlated.

\section{ACKNOWLEDGMENT}

The authors would like to thank David Whitley (Statnett SF) for his thorough comments.

\section{REFERENCES}

[1] M. de la Torre Rodríguez, M. Scherer, D. Whitley, and F. Reyer, "Frequency containment reserves dimensioning and target performance in the european power system," in IEEE PES General Meeting Conference Exposition, July 2014, pp. 1-5.

[2] ENTSO-E, "Network Code on Electricity Balancing (Version 3.0)," www.entsoe.eu, August 2014.

[3] M. Scherer, M. Zima, and G. Andersson, "An integrated pan-European ancillary services market for frequency control," Energy Policy, vol. 62, pp. 292-300, 2013.

[4] S. Sterpu and M. Tuan, "Sharing frequency response between asynchronous electrical systems," in IEEE Power Energy Society General Meeting, July 2009, pp. 1-6.

[5] G. Fujita, G. Shirai, and R. Yokoyama, "Automatic generation control for DC-link power system," in IEEE/PES Transmission and Distribution Conference and Exhibition 2002: Asia Pacific, vol. 3, October 2002, pp. 1584-1588.

[6] M. Sanpei, A. Kakehi, and H. Takeda, "Application of multi-variable control for automatic frequency controller of HVDC transmission system," IEEE Transactions on Power Delivery, vol. 9, no. 2, pp. 10631068, April 1994.

[7] M. Davies, A. Kolz, M. Kuhn, D. Monkhouse, and J. Strauss, "Latest control and protection innovations applied to the Basslink HVDC interconnector," in The 8th IEE International Conference on AC and DC Power Transmission (ACDC 2006), March 2006, pp. 30-35.

[8] OpHB-Team, "Policy 1: Load-Frequency Control and Performance," in UCTE Operation Handbook, March 2009.

[9] M. Scherer, E. Iggland, A. Ritter, and G. Andersson, "Improved frequency bias factor sizing for non-interactive control," in Cigré 2012 Session, C2-113, 2012.

[10] P. Zolotarev, M. Gökeler, M. Kuring, H. Neumann, and E.-M. Kurscheid, "Grid Control Cooperation - A Framework for Technical and Economical Cross-Border Optimization for Load-Frequency Control," in Cigré 2012 Session, C2-107, 2012. 